\title{
Enantioselective Catalysis of the Aza-Cope Rearrangement by a Chiral Supramolecular Assembly
}

\author{
Casey J. Brown, Robert G. Bergman, ${ }^{*}$ and Kenneth N. Raymond* \\ Department of Chemistry, University of California, Berkeley, CA 94720-1460; and Division of Chemical Sciences, Lawrence \\ Berkeley National Laboratory, Berkeley, CA 94720
}

RECEIVED DATE (automatically inserted by publisher); rbergman@berkeley.edu, raymond@socrates.berkeley.edu

Nanoscale molecular flasks have increasingly been used to promote novel reactivity or impart powerful selectivity through precise, noncovalent interactions with substrate molecules. Encapsulation of substrate molecules within these host structures may stabilize reactive species ${ }^{1}$ or, conversely, promote substrate reactivity. ${ }^{2}$ The confined environment within these supramolecular hosts has also been demonstrated to impart remarkable size and shape selectivity. ${ }^{3}$

Employing these supramolecular assemblies in asymmetric catalysis remains an important challenge. ${ }^{4}$ Chiral building blocks may be used to construct chiral supramolecular assemblies, in some cases generating additional elements of chirality during the assembly of these structures. While chiral supramolecular assemblies have been shown to carry out enantioselective stoichiometric reactions, or catalyze reactions with modest ee, a highly enantioselective catalytic transformation has not yet been demonstrated. ${ }^{5}$ We now report such catalysis.
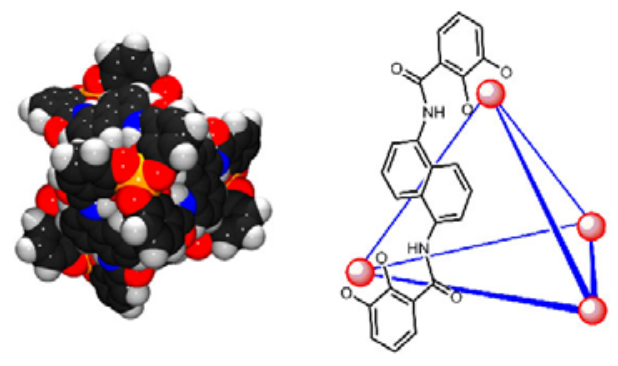

Figure 1. (Left) Space-filling model of $\left[\mathrm{Ga}_{4} \mathrm{~L}_{6}\right]^{12-}$ assembly 1 , sighted down the 3 -fold axis. (Right) Schematic of assembly 1 . Only one ligand is shown for clarity.

Raymond and coworkers have developed $\left[\mathrm{Ga}_{4} \mathrm{~L}_{6}\right]^{12-}$ assembly $\mathbf{1}$, a self-assembling supramolecular structure. ${ }^{6}$ In collaboration with the Bergman group, this cluster has been shown capable of catalyzing a variety of chemical transformations with low catalyst loadings and enzyme-like kinetics, including the aza-Cope rearrangement and the hydrolysis of orthoformates and acetals. ${ }^{7}$ Importantly, $\mathbf{1}$ is chiral due to the three bidentate catecholates coordinating each gallium center (Figure 1). Mechanical coupling between the four vertices enforces the same helical configuration $\left(\Delta\right.$ or $\Lambda$ ) at each metal center. ${ }^{8}$ As a result, two enantiomeric forms of $\mathbf{1}$ exist, $\Delta \Delta \Delta \Delta$ and $\Lambda \Lambda \Lambda \Lambda$. Though $\mathbf{1}$ is synthesized as the racemate, addition of (-)- $N^{\prime}$-methylnicotinium iodide (S-nicI) causes the spontaneous resolution of the two enantiomers, allowing access to pure $\Delta \Delta \Delta \Delta$-(S-nic $\subset \mathbf{1}$, where $\subset$ denotes encapsulation) and pure $\Lambda \Lambda \Lambda \Lambda$-(S-nic $\subset \mathbf{1})$. Ion exchange chromatography allows isolation of each enantiomer as the tetramethylammonium salt. ${ }^{9}$

For this study, the aza-Cope rearrangement of enammonium substrates was selected (Figure 2) to evaluate $\mathbf{1}$ as an enantioselective catalyst. ${ }^{9 c}$ Encapsulation of enammonium substrates within $\mathbf{1}$ enforces a reactive conformation. The product iminium ions are vulnerable to hydrolysis, producing neutral aldehydes which are not encapsulated in $\mathbf{1}$. As long as $\mathrm{R}_{1} \neq \mathrm{R}_{2}$, the rearrangement generates a chiral center and potentially enantioselective within chiral assembly $\mathbf{1}$. Since obtaining suitable quantities of enantiopure $K_{12} \mathbf{1}$ is not practical, reactivity compatible with $\left(\mathrm{NMe}_{4}\right)_{12} \mathbf{1}$ is required. . Enammonium substrates $\mathbf{2}$ are more tightly bound than $\mathrm{NMe}_{4}{ }^{+}$, enabling efficient catalysis within $\left(\mathrm{NMe}_{4}\right)_{12} \mathbf{1}$. We describe here the application of enantiopure $\mathbf{1}$ in catalyzing the aza-Cope rearrangement, achieving enantioselectivities for host-guest catalysis that are remarkable in view of the fact that the cavity bears no reactive functional groups. 


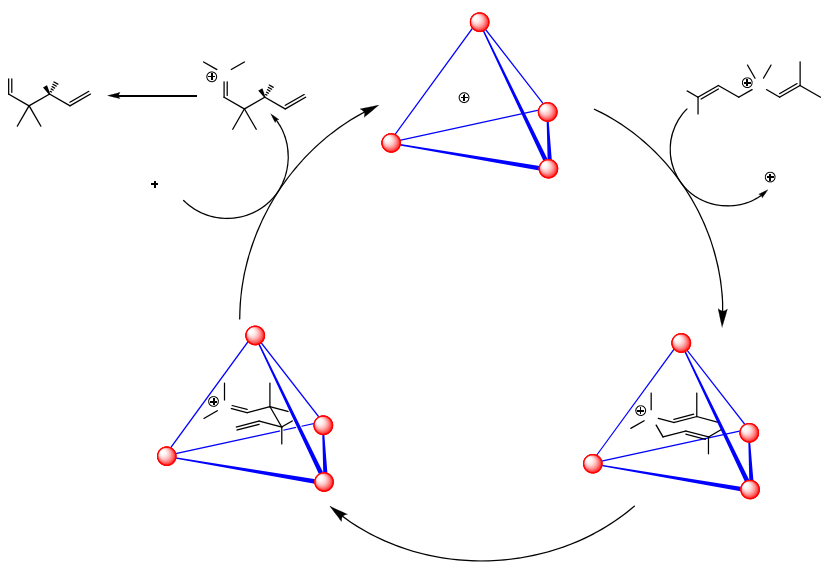

Figure 2. Catalytic cycle for the aza-Cope rearrangement within supramolecular assembly $\mathbf{1 .}$

A series of prochiral enammonium tosylates (2a-g) were treated with catalytic amounts of $\left(\mathrm{NMe}_{4}\right)_{12}[\Delta \Delta \Delta \Delta-\mathbf{1}]$ to explore the possibility of asymmetric induction in the host-catalyzed aza-Cope rearrangement. High catalyst loadings were used to avoid precipitation of the catalyst-substrate complexes. Lower catalyst loadings (3\%) could be used in a mixed MeOH/DMSO solvent system, with identical yields and selectivities. Slower reaction times and difficulty separating the products from the reaction mixtures, however, made this less desirable. The chiral product aldehydes were extracted into $\mathrm{d}_{8}$-toluene solution and were analyzed by chiral GC (Figure 3). Enantioselectivities above 60\% were observed for the cis-ethyl (2b) and trans-isopropyl (2f) salts (Table 1).

The observed enantioselectivities displayed large variation with subtle changes in substrate size and shape. The enantioselectivity obtained with substrate $\mathbf{2 b}(64 \%$ ee) erodes rapidly with addition of a single carbon (2d, $9 \%$ ee) or changing the geometry of the double bond $(\mathbf{2 c}, 25 \%$ ee). The shape selectivity is further exemplified by the different selectivities observed for isopropyl-substituted substrate 2f, which exhibits much higher enantioselectivity than $n$-propyl-substituted substrate $2 \mathbf{e}^{10}$

Table 1. Evaluation of Asymmetric Induction in the Aza-Cope Rearrangement Catalyzed by 1.

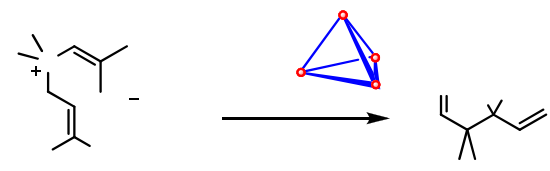

\begin{tabular}{ccccc}
\hline Substrate & $\mathrm{R}_{1}$ & $\mathrm{R}_{2}$ & ${\text { Yield }(\%)^{a}}^{a}$ & ee \\
\hline $2 \mathrm{a}$ & $\mathrm{H}$ & $\mathrm{Me}$ & 45 & 0 \\
$2 \mathrm{~b}$ & $\mathrm{Et}$ & $\mathrm{H}$ & 58 & 64 \\
$2 \mathrm{c}$ & $\mathrm{H}$ & $\mathrm{Et}$ & 69 & 25 \\
$2 \mathrm{~d}$ & $\mathrm{Pr}$ & $\mathrm{H}$ & 68 & 9 \\
$2 \mathrm{e}$ & $\mathrm{H}$ & $\mathrm{Pr}$ & 21 & 23 \\
$2 \mathrm{f}$ & $\mathrm{H}$ & $\mathrm{iPr}$ & 74 & 60 \\
$2 \mathrm{f}^{\mathrm{b}}$ & $\mathrm{H}$ & $\mathrm{iPr}$ & 49 & 78 \\
$2 \mathrm{~g}^{c}$ & $\mathrm{H}$ & $\mathrm{nBu}$ & 82 & 6 \\
\hline
\end{tabular}

${ }^{\mathrm{a}}$ Yields measured by ${ }^{1} \mathrm{H} \mathrm{NMR}$ with $\mathrm{CHCl}_{3}$ as an internal standard. ${ }^{b}$ Reaction conducted at $5{ }^{\circ} \mathrm{C}$ over 8 days. ${ }^{\mathrm{c}} 0 \%$ catalyst loading.

Conducting the rearrangement at lower temperatures improves the enantioselectivity. Using substrate $\mathbf{2 f}$ the rearrangement was tested down to $5{ }^{\circ} \mathrm{C}$. Lower temperatures result in lower yields and extended reaction times, but the enantioselectivities improve, up to $78 \%$ ee.

These enantioselectivities are much higher than the product binding diastereoselectivities (de's) observed earlier using racemic 1. In the absence of $\mathrm{NMe}_{4}{ }^{+}$cations, the product iminium ion concentrations can build up without being hydrolyzed. For the prochiral substrates $\mathbf{2 a}-$ $\mathbf{2 g}$, none of the product iminium ions is encapsulated in racemic $\mathbf{1}$ with de greater than $20 \% .^{7 \mathrm{c}}$ Chiral discrimination by $\mathbf{1}$ is therefore much stronger than that of product binding alone. ${ }^{11}$

To gain insight into the basis for enantioselectivity in this transformation, torsional sampling of substrate $\mathbf{2 f}$ was carried out within the cavity of $\Delta \Delta \Delta \Delta-\mathbf{1}$ (Figure 3). The prochiral carbon bearing the isopropyl substituent is in each case directed towards one of the four helically chiral vertices of $\mathbf{1}$. Close contact with the chiral element of the host may be responsible for the selectivity of the rearrangement. The two calculated structures differ in energy by $2.2 \mathrm{kcal} / \mathrm{mol}$, appropriate to the degree of selectivity observed in the transformation neglecting transition-state effects.

Pericyclic reactions present a special challenge for asymmetric catalysis. Ordinarily, coordinating groups on the substrate are required, driving complexation of a chiral Lewis acid. ${ }^{12}$ Assembly $\mathbf{1}$ is able to render this pericyclic reaction enantioselective only by confining the reaction to a chiral space, rather than interacting specifically with a moiety on the substrate. This is an attractive feature of supramolecular reaction vessels and is an important virtue of this complementary catalytic strategy.

The enantioselectivity achieved here $(78 \%$ ee) is the highest observed from catalysis by a synthetic chiral supramolecular host to date. While the cavity of $\mathbf{1}$ is primarily bounded by rigid, achiral naphthalenes, the helically chiral metal centers produce good asymmetric induction in this rearrangement. As with enzymes, precise structural control of the active site is achieved indirectly through noncovalent interactions. These results demonstrate the promise of using chiral supramolecular assemblies in asymmetric catalysis. 

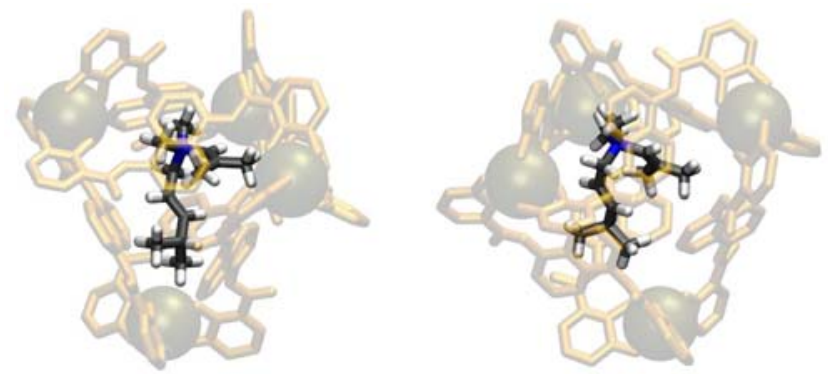

Figure 3. Prochiral conformations of substrate $2 \mathbf{f}$ within $\Delta \Delta \Delta \Delta-\mathbf{1}$, modeled by torsional sampling in the OPLS_2005 force field. The structure at the right is predicted to be $2.2 \mathrm{kcal} / \mathrm{mol}$ higher in energy. Animations of these structures are included in the Supporting Information for clarity.

Acknowledgement. We thank Dr. Mike Pluth, Courtney Hastings, and Jeff Mugridge for helpful discussions. This work was supported by the Director, Office of Science, Office of Basic Energy Sciences, and the Division of Chemical Sciences, Geosciences, and Biosciences of the U.S. Department of Energy at LBNL under Contract No. DE-AC02-05CH11231.

Supporting Information Available. Experimental procedures, structural proofs, and spectral data for all new compounds are provided (5 pages) (PDF). This material is available free of charge via the internet at http://pubs.acs.org.

(1) (a) Iwasawa, T.; Hooley, R. J.; Rebek, J. Science 2007, 317, 493-496. (b) Dong, V. M.; Fiedler, D.; Carl, B.; Bergman, R. G.; Raymond, K. N. J. Am. Chem. Soc. 2006 128, 14464-14465. (c) Brumaghim, J. L.; Michels, M.; Raymond, K. N. Eur. J. Org. Chem. 2004, 4552-4559. (d) M. Kuil, T. Soltner, P. W. N. M. Van Leeuwen and J. N. H. Reek, J. Am. Chem. Soc., 2006, 128, 11344.

(2) (a) Yoshizawa, M.; Tamura, M.; Fujita, M. Science 2006, 312, 251-254. (b) Pluth, M. D.; Bergman, R. G.; Raymond, K. N. Science 2007, 316, 85-88. (c) Hou, J. L.; Ajami, D.; Rebek, J. J. Am. Chem. Soc. 2008, 130, 7810-7811.

(3) (a) Leung, D. H.; Bergman, R. G.; Raymond, K. N. J. Am. Chem. Soc. 2007, 129, 2746-2747. (b) Leung, D. H.; Bergman, R. G.; Raymond, K. N. J. Am. Chem. Soc. 2006, 128, 9781-9797. (c) Seneque, O.; Rager, M. N.; Giorgi, M.; Reinaud, O. J. Am. Chem. Soc. 2000, 122, 6183-6189. (d) Natarajan, A.; Kaanumalle, L. S.; Jockusch, S.; Gibb, C. L. D.; Gibb, B. C.; Turro, N. J.; Ramamurthy, V. J. Am. Chem. Soc. 2007, 129, 4132-4133.

(4) (a) Hembury, G. A.; Borovkov, V. V.; Inoue, Y. Chem. Rev. 2008, 108, 1-73. (b) Koblenz, T. S.; Wassenaar, J.; Reek, J. N. H.; Chem. Soc. Rev. 2008, 37, 247-262.

(5) (a) Lu, R.; Yang, C.; Cao, Y.; Tong, L.; Jiao, W.; Wada, T.; Wang, Z.; Mori, T.; Inoue, Y. J. Org. Chem. 2008, 73, 7695-7701. (b) Nishioka, Y.; Yamaguchi, T.; Kawano, M.; Fujita, M. J. Am. Chem. Soc. 2008, 130, 8160-8161. (c) Lee, S. J.; Cho, S.-H.; Mulfort, K. L.; Tiede, D. M.; Hupp, J. T. Nguyen, S. T.; J. Am. Chem. Soc. 2008, 130, 16828-16829. (d) Yang, C.; Mori, T.; Inoue, Y.; J Org. Chem. 2008, 73, 5876. (e) Origane, Y.; Ko, Y.H.;Selvapalam, N.; Kim, K.; Inoue, Y.; 2008, $130,8574$.

(6) Caulder, D.; Raymond, K. N. J. Chem. Soc., Dalton Trans., 1999, 8, 1185-1200.

(7) Pluth, M. D.; Bergman, R. G.; Raymond, K. N. Angew. Chem., Int. Ed. 2007, 46, 8587-8589. (b) Pluth, M. D.; Bergman, R. G.; Raymond, K. N. J. Am. Chem. Soc. 2008, 130, 11423-11429. (c) Fiedler, D.; van Halbeek, H.; Bergman, R. G.; Raymond, K. N. J. Am. Chem. Soc. 2006, 128, 10240-10252. (d) Hastings, C. J.; Fiedler, D.; Bergman, R. G.; Raymond, K. N. J. Am. Chem. Soc. 2008, 130, 10977-10983.

(8) Ziegler, M.; Davis, A. V.; Johnson, D. W.; Raymond, K. N. Angew. Chem., Int. Ed. 2003, 42, 665-668.

(9) Davis, A. V.; Fiedler, D.; Ziegler, M.; Terpin, A.; Raymond, K. N. J. Am. Chem. Soc. 2007, 129, 15354-15363.

(10) High shape specificity in chiral recognition by $\mathbf{1}$ has also been observed in the C-H activation of aldehydes by prochiral iridium half-sandwich complexes (ref. 5 a) but the earlier system was not catalytic and was not designed to allow isolation of enantioencriched product.

(11) Low diastereoselectivity observed in racemic 1 (ref. 7c) should be attributed to equilibration to a lower thermodynamic selectivity of product binding. Rapid exchange of the product iminium ions masks the enantioselectivity of the rearrangement when using racemic $\mathrm{Ga}_{4} \mathrm{~L}_{6}$.

(12) Hiersemann, M.; Abraham, L. Eur. J. Org. Chem. 2002, 1461-1471. 